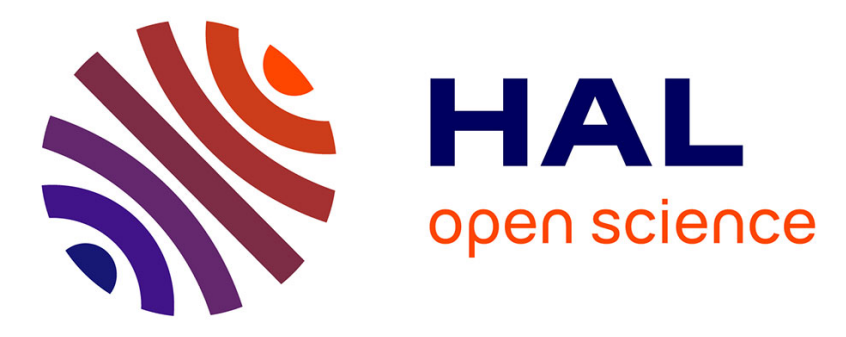

\title{
The Green-Kubo formula for locally interacting open fermionic systems
}

\author{
Vojkan Jaksic, Yoshiko Ogata, Claude-Alain Pillet
}

\section{To cite this version:}

Vojkan Jaksic, Yoshiko Ogata, Claude-Alain Pillet. The Green-Kubo formula for locally interacting open fermionic systems. Annales Henri Poincaré, 2007, 8 (6), pp.1013-1036. 10.1007/s00023-0070327-7 . hal-00090544v2

\section{HAL Id: hal-00090544 \\ https://hal.science/hal-00090544v2}

Submitted on 4 Jan 2007

HAL is a multi-disciplinary open access archive for the deposit and dissemination of scientific research documents, whether they are published or not. The documents may come from teaching and research institutions in France or abroad, or from public or private research centers.
L'archive ouverte pluridisciplinaire HAL, est destinée au dépôt et à la diffusion de documents scientifiques de niveau recherche, publiés ou non, émanant des établissements d'enseignement et de recherche français ou étrangers, des laboratoires publics ou privés. 


\title{
The Green-Kubo formula for locally interacting fermionic open systems
}

\author{
V. Jakšić ${ }^{1}$, Y. Ogata ${ }^{2,3}$, C.-A. Pillet ${ }^{4}$ \\ ${ }^{1}$ Department of Mathematics and Statistics \\ McGill University \\ 805 Sherbrooke Street West \\ Montreal, QC, H3A 2K6, Canada \\ ${ }^{2}$ Department of Mathematics, University of California, Davis \\ Davis, CA 95616, USA \\ ${ }^{3}$ Department of Mathematical Sciences \\ University of Tokyo \\ Komaba,Tokyo,153-8914 Japan \\ ${ }^{4}$ FRUMAM \\ CPT-CNRS, UMR 6207 \\ Université du Sud, Toulon-Var, B.P. 20132 \\ 83957 La Garde Cedex, France \\ January 4, 2007
}

\begin{abstract}
We consider a model describing finitely many free Fermi gas reservoirs coupled by local interactions and prove the Green-Kubo formulas and the Onsager reciprocity relations for heat and charge fluxes generated by temperature and chemical potential differentials.
\end{abstract}




\section{Introduction}

This is the fourth in a series of papers [JOP1, JOP2, JOP3] dealing with derivation of Green-Kubo formulas (GKF) and Onsager reciprocity relations (ORR) in quantum statistical mechanics. The first two papers [JOP1, JOP2] were devoted to the abstract axiomatic derivation of GKF and ORR for open systems driven by thermodynamical forces associated to temperature and chemical potential differentials. This paper and [JOP3] are devoted to the study of concrete models.

In [JOP3] we have studied the well-known spin-fermion model describing the interaction of an $N$-level atom with finitely many independent free Fermi gas reservoirs [Da, LeSp, JP2]. Combining the results of [JOP1, JOP2] with spectral theory of non-equilibrium steady states developed in [JP2] we have established GKF and ORR for this class of models.

In this paper we study a model describing finitely many free Fermi gas reservoirs coupled by local interactions and show that the abstract derivation of [JOP1, JOP2] combined with scattering theory of non-equilibrium steady states (see [BM1, AM, BM2, Ru1, FMU]) yields the GKF and ORR for this class of models.

Throughout the paper we shall assume that the reader is familiar with general aspects of linear response theory discussed in [JOP1, JOP2, JOP3] and with the algebraic formalism of quantum statistical mechanics [BR1, BR2]. A modern introduction to these topics can be found in [JP3, FMU] and in the recent lecture notes [AJPP1].

The paper is organized as follows. In Subsection 1.1 for notational purposes we review the description of a free Fermi gas in the algebraic formalism of quantum statistical mechanics. In Subsection 1.2 we introduce the model and state our results. The strategy of the proof is the same as in [JOP3] and is described in Section 3.1. This strategy reduces the proof of all our results to a technical estimate formulated in Theorem 3.1. This estimate, which is our main technical result, is established in Section 3.2.

Acknowledgment. The research of V.J. was partly supported by NSERC. A part of this work has been done during V.J.'s visit to CPT-CNRS and during his stay as a Forchheimer Visiting Professor at The Hebrew University of Jerusalem. He would like to thank H. Farkas and Y. Last for the hospitality of the Einstein Institute of Mathematics at The Hebrew University. The research of Y.O. was supported by the Japan Society for the Promotion of Science. A part of this work has been done during the stay of Y.O. at CPT-CNRS, partly supported by the Canon Foundation in Europe and JSPS.

\subsection{Preliminaries}

Let $\mathfrak{h}$ and $h_{0}$ be given Hilbert space and Hamiltonian. The corresponding free Fermi gas is described by the $C^{*}$-dynamical system $\left(\mathcal{O}, \tau_{0}\right)$ where:

(i) $\mathcal{O}=\operatorname{CAR}(\mathfrak{h})$ is the CAR algebra over $\mathfrak{h}$. We denote by $a^{*}(f) / a(f)$ the creation/annihilation operator associated to $f \in \mathfrak{h}$. As usual, $a^{\#}$ stands for either $a$ or $a^{*}$;

(ii) $\tau_{0}^{t}$ is the group of Bogoliubov $*$-automorphisms generated by $h_{0}, \tau_{0}^{t}\left(a^{\#}(f)\right)=a^{\#}\left(\mathrm{e}^{\mathrm{i} t h_{0}} f\right)$. We denote by $\delta_{0}$ the generator of $\tau_{0}$;

The gauge group of the free Fermi gas is the group of Bogoliubov $*$-automorphisms $\vartheta^{\varphi}, \varphi \in \mathbb{R}$, generated by the identity operator on $\mathfrak{h}$. The physical observables are gauge invariant and hence elements of

$$
\mathcal{O}_{\vartheta}=\left\{A \in \mathcal{O} \mid \vartheta^{\varphi}(A)=A \text { for all } \varphi \in \mathbb{R}\right\} .
$$

$\mathcal{O}_{\vartheta}$ is the $\tau_{0}$-invariant $C^{*}$-subalgebra of $\mathcal{O}$ generated by $\left\{a^{*}(f) a(g) \mid f, g \in \mathfrak{h}\right\}$ and $\mathbb{1}$.

Let $\beta>0$ and $\mu \in \mathbb{R}$ be parameters and $\omega_{\beta \mu}$ the gauge-invariant quasi-free state on $\mathcal{O}$ generated by

$$
T_{\beta \mu}=\frac{1}{1+\mathrm{e}^{\beta\left(h_{0}-\mu\right)}} .
$$


The quantum dynamical system $\left(\mathcal{O}, \tau_{0}, \omega_{\beta \mu}\right)$ describes a free Fermi gas in thermal equilibrium at inverse temperature $\beta$ and chemical potential $\mu$. We remark that $\omega_{\beta \mu}$ is the unique $\beta$-KMS state for the $C^{*}$-dynamics $\tau_{0}^{t} \circ \vartheta^{-\mu t}$ and that $\omega_{\beta \mu} \uparrow \mathcal{O}_{\vartheta}$ is a $\left(\tau_{0}, \beta\right)$-KMS state on $\mathcal{O}_{\vartheta}$.

Let $V \in \mathcal{O}_{\vartheta}$ be a self-adjoint perturbation and $\tau_{\lambda}$ the perturbed $C^{*}$-dynamics generated by $\delta_{\lambda}=\delta_{0}+\mathrm{i} \lambda[V, \cdot]$ where $\lambda \in \mathbb{R}$ is a coupling constant. We recall that for $A \in \mathcal{O}$ and $t \geq 0$,

$$
\tau_{\lambda}^{t}(A)=\tau_{0}^{t}(A)+\sum_{n=1}^{\infty}(\mathrm{i} \lambda)^{n} \int_{0 \leq s_{n} \leq \cdots \leq s_{1} \leq t}\left[\tau_{0}^{s_{n}}(V),\left[\cdots,\left[\tau_{0}^{s_{1}}(V), \tau_{0}^{t}(A)\right] \cdots\right]\right] \mathrm{d} s_{1} \cdots \mathrm{d} s_{n} .
$$

In this paper we shall consider self-adjoint perturbations of the form

$$
V=\sum_{k=1}^{K} \prod_{j=1}^{n_{k}} a^{*}\left(u_{k j}\right) a\left(v_{k j}\right)
$$

where $K$ and $n_{k}$ 's are finite. We set $\bar{n}=\max _{k} n_{k}$. Denote $\mathcal{D}_{0}=\left\{u_{k j}, v_{k j}\right\}$. By rescaling $\lambda$, without loss of generality we may assume that

$$
\max _{f \in \mathcal{D}_{0}}\|f\|=1
$$

If $\bar{n}=1$, then $\tau_{\lambda}^{t}\left(a^{\#}(f)\right)=a^{\#}\left(\mathrm{e}^{\mathrm{i} t h_{\lambda}} f\right)$ where $h_{\lambda}=h_{0}+\lambda \sum_{k}\left(v_{k}, \cdot\right) u_{k}$, and so the $C^{*}$-dynamics $\tau_{\lambda}$ is also a group of Bogoliubov $*$-automorphisms. This special case is exactly solvable and has been studied in detail in [AJPP2] (for additional information and references about quasi-free open quantum systems we refer the reader to recent lecture notes [AJPP1, JKP]).

The following technical result will play a key role in our paper.

Theorem 1.1 Let $A=a^{\#}\left(f_{1}\right) \cdots a^{\#}\left(f_{m}\right)$ be a monomial of order $m$ and

$$
\mathcal{C}_{A}^{(n)}\left(s_{0}, \ldots, s_{n}\right)=\left[V,\left[\tau_{0}^{s_{n}}(V),\left[\cdots,\left[\tau_{0}^{s_{1}}(V), \tau_{0}^{s_{0}}(A)\right] \cdots\right]\right]\right]
$$

Then for all $n \geq 0$ there exist a finite index set $\mathcal{P}_{n}(A)$, monomials $F_{A, p}^{(n)} \in \mathcal{O}$, and scalar functions $G_{A, p}^{(n)}$ such that

$$
\mathcal{C}_{A}^{(n)}\left(s_{0}, \ldots, s_{n}\right)=\sum_{p \in \mathcal{P}_{n}(A)} G_{A, p}^{(n)}\left(s_{0}, \ldots, s_{n}\right) F_{A, p}^{(n)}\left(s_{0}, \ldots, s_{n}\right) .
$$

\section{Moreover,}

1. The order of the monomial $F_{A, p}^{(n)}$ does not exceed $2(n+1)(\bar{n}-1)+m$.

2. The factors of $F_{A, p}^{(n)}$ are from

$$
\left\{a^{\#}\left(\mathrm{e}^{\mathrm{i} s h_{0}} g\right) \mid g \in \mathcal{D}_{0}, s \in\left\{0, s_{1}, \ldots, s_{n}\right\}\right\} \cup\left\{a^{\#}\left(\mathrm{e}^{\mathrm{i} s_{0} h_{0}} g\right) \mid g \in \mathcal{A}\right\},
$$

where $\mathcal{A}=\left\{f_{1}, \cdots, f_{m}\right\}$. The number of factors from the first set does not exceed $(n+1)(2 \bar{n}-1)$ while the number of factors from the second set does not exceed $m-1$.

3. Suppose that

$$
\ell=\int_{0}^{\infty} \sup _{f \in \mathcal{D}_{0}, g \in \mathcal{D}_{0} \cup \mathcal{A}}\left|\left(f, \mathrm{e}^{i t h_{0}} g\right)\right| \mathrm{d} t<\infty
$$

denote

$$
\ell_{0}=\int_{0}^{\infty} \sup _{f, g \in \mathcal{D}_{0}}\left|\left(f, \mathrm{e}^{\mathrm{i} s h_{0}} g\right)\right| \mathrm{d} s
$$


and set

$$
\Lambda_{0}= \begin{cases}\frac{1}{2 K \ell_{0}} & \text { if } \bar{n}=1, \\ \frac{1}{2 \bar{n} K \ell_{0}} \frac{(2 \bar{n}-2)^{2 \bar{n}-2}}{(2 \bar{n}-1)^{2 \bar{n}-1}} & \text { if } \bar{n}>1 .\end{cases}
$$

If $\bar{n}=1$ and $|\lambda|<\Lambda_{0}$ or if $\bar{n}>1$ and $|\lambda| \leq \Lambda_{0}$ then the sum

$$
W=\sum_{n=0}^{\infty}|\lambda|^{n+1} \sum_{p \in \mathcal{P}_{n}(A)} \int_{0 \leq s_{n} \leq \cdots \leq s_{0}<\infty}\left|G_{A, p}^{(n)}\left(s_{0}, \ldots, s_{n}\right)\right| \mathrm{d} s_{0} \cdots \mathrm{d} s_{n}
$$

is finite and satisfies

$$
W \leq\left(1+\frac{2 \bar{n} K \ell|\lambda|}{\left(1-|\lambda| / \Lambda_{0}\right)+2 \bar{n}(2 \bar{n}-2) K \ell_{0}|\lambda|}\right)^{m}-1
$$

Remark 1. Parts (1) and (2) of this theorem are easy to prove and are stated for reference purpose. The key fact is Part (3) which we shall prove using the fundamental Botvich-Gută -Maassen integral estimate [BGM]. Related but weaker results can be obtained using the integral estimates of [BM1, BM2, FMU].

Remark 2. In our applications we shall not need the explicit form of the bound (1.5).

Our first regularity assumption is

(A1) There exists a dense vector subspace $\mathcal{D} \subset \mathfrak{h}$ such that $\mathcal{D}_{0} \subset \mathcal{D}$ and that the functions

$$
\mathbb{R} \ni t \mapsto\left(f, \mathrm{e}^{\mathrm{i} t h_{0}} g\right),
$$

are in $L^{1}(\mathbb{R}, \mathrm{d} t)$ for all $f, g \in \mathcal{D}$.

Note that this assumption implies that $h_{0}$ has purely absolutely continuous spectrum.

A consequence of Theorem 1.1 is

Theorem 1.2 Assume that (A1) holds and that $|\lambda|<\Lambda_{0}$. Then the limits

$$
\gamma_{\lambda}^{+}(A)=\lim _{t \rightarrow+\infty} \tau_{0}^{-t} \circ \tau_{\lambda}^{t}(A)
$$

exist for all $A \in \mathcal{O}$ and define a $*$-automorphism $\gamma_{\lambda}^{+}: \mathcal{O} \rightarrow \mathcal{O}$.

Remark. Under additional regularity assumptions one can also obtain information about the rate of convergence in (1.6), see [JP4] for details.

Although Theorem 1.2 is a well-known result (see [Ro, BM1, BM2, FMU]), for the reader convenience we will sketch its proof in Subsection 2.2.

\subsection{The model and the result}

Our starting point are finitely many, say $M$, independent free Fermi gasses $\mathcal{R}_{j}$ in equilibrium at inverse temperatures $\beta_{j}>0$ and chemical potentials $\mu_{j} \in \mathbb{R}$. More precisely, $\mathcal{R}_{j}$ is described by the quantum dynamical system $\left(\mathcal{O}_{j}, \tau_{j}, \omega_{j}\right)$ where:

(i) $\mathcal{O}_{j}=\operatorname{CAR}\left(\mathfrak{h}_{j}\right)$ is the CAR algebra over the single fermion Hilbert space $\mathfrak{h}_{j}$;

(ii) $\tau_{j}^{t}$ is the group of Bogoliubov $*$-automorphisms generated by the single fermion Hamiltonian $h_{j}$; 
(iii) $\omega_{j}$ is the gauge-invariant quasi-free state generated by

$$
T_{j}=\frac{1}{1+\mathrm{e}^{\beta_{j}\left(h_{j}-\mu_{j}\right)}} .
$$

We denote by $\vartheta_{j}$ the gauge group of $\mathcal{R}_{j}$. The generators of $\tau_{j}$ and $\vartheta_{j}$ are denoted by $\delta_{j}$ and $\xi_{j}$.

Let

$$
\mathfrak{h}=\bigoplus_{j=1}^{M} \mathfrak{h}_{j}, \quad h_{0}=\bigoplus_{j=1}^{M} h_{j}, \quad T=\bigoplus_{j=1}^{M} T_{j}
$$

The joint system $\mathcal{R}=\sum \mathcal{R}_{j}$ in absence of interaction is described by the quantum dynamical system $\left(\mathcal{O}, \tau_{0}, \omega\right)$, where $\mathcal{O}=\operatorname{CAR}(\mathfrak{h}), \tau_{0}^{t}$ is the group of Bogoliubov $*$-automorphisms generated by $h_{0}$, and $\omega$ is the gauge-invariant quasi-free state generated by $T$. We denote by $\delta_{0}$ the generator of $\tau_{0}$ and by $\xi$ the generator of the gauge group $\vartheta$ of the joint system. Obviously, $\delta_{0}=\sum_{j} \delta_{j}$ and $\xi=\sum_{j} \xi_{j}$.

Let $V \in \mathcal{O}_{\vartheta}$ be a perturbation of the form (1.2). This perturbation describes the coupling of the reservoirs, and, possibly, self-interactions within the reservoirs. Let $\lambda \in \mathbb{R}$ be a coupling constant and $\tau_{\lambda}$ the $C^{*}$-dynamics on $\mathcal{O}$ generated by $\delta_{\lambda}=\delta_{0}+\mathrm{i} \lambda[V, \cdot]$. The interacting joint system is described by the quantum dynamical system $\left(\mathcal{O}, \tau_{\lambda}, \omega\right)$.

Let $\gamma_{\lambda}^{+}$be as in Theorem 1.2 and $\omega_{\lambda+}=\omega \circ \gamma_{\lambda}^{+}$. A consequence of Theorem 1.2 (see Subsection 2.2) is:

Theorem 1.3 Assume that (A1) holds and that $|\lambda|<\Lambda_{0}$. Then for all $\omega$-normal states $\eta$ and $A \in \mathcal{O}$,

$$
\lim _{t \rightarrow+\infty} \eta \circ \tau_{\lambda}^{t}(A)=\omega_{\lambda+}(A) .
$$

The state $\omega_{\lambda+}$ is the NESS of the quantum dynamical system $\left(\mathcal{O}, \tau_{\lambda}, \omega\right)$ [Ru1, JP3]. Clearly, this NESS depends on $\beta_{j}$ and $\mu_{j}$.

Let $\beta_{\text {eq }}>0$ and $\mu_{\text {eq }} \in \mathbb{R}$ be given (equilibrium) values of the inverse temperature and chemical potential. We are interested in linear response of $\mathcal{R}$ to thermodynamical forces

$$
X_{j}=\beta_{\mathrm{eq}}-\beta_{j}, \quad Y_{j}=\beta_{j} \mu_{j}-\beta_{\mathrm{eq}} \mu_{\mathrm{eq}} .
$$

Let $X=\left(X_{1}, \cdots, X_{M}\right), Y=\left(Y_{1}, \cdots, Y_{M}\right)$. We indicate the dependence on $X, Y$ by denoting

$$
\omega_{X Y}=\omega, \quad \omega_{\lambda X Y+}=\omega_{\lambda+}, \quad T_{X Y}=T .
$$

Note that by Araki perturbation theory $\omega_{\lambda 00+}$ is the unique $\beta_{\mathrm{eq}}-\mathrm{KMS}$ state for the $C^{*}$-dynamics $\tau_{\lambda}^{t} \circ \vartheta^{-\mu_{\mathrm{eq}} t}$. We denote this state by $\omega_{\lambda \text { eq }}$.

In what follows we shall assume:

(A2) The operators $h_{j}$ are bounded.

Although our method of proof extends to unbounded $h_{j}$ 's (see Remark 2 after Theorem 1.5), the above assumption covers most cases of physical interest to which our results apply and allows for technically simpler exposition of the proofs.

The observables describing the heat and charge flux out of $\mathcal{R}_{j}$ are

$$
\Phi_{j}=\lambda \delta_{j}(V), \quad \mathcal{J}_{j}=\lambda \xi_{j}(V) .
$$

Clearly, $\Phi_{j}, \mathcal{J}_{j} \in \mathcal{O}_{\vartheta}$. The conservation laws

$$
\sum_{j=1}^{M} \omega_{\lambda X Y+}\left(\Phi_{j}\right)=0, \quad \sum_{j=1}^{M} \omega_{\lambda X Y+}\left(\mathcal{J}_{j}\right)=0
$$


hold. The entropy production of the NESS $\omega_{\lambda X Y+}$ is defined by

$$
\operatorname{Ep}\left(\omega_{\lambda X Y+}\right)=\omega_{\lambda X Y+}\left(-\sum_{j=1}^{M} \beta_{j}\left(\Phi_{j}-\mu_{j} \mathcal{J}_{j}\right)\right)=\sum_{j=1}^{M} X_{j} \omega_{\lambda X Y+}\left(\Phi_{j}\right)+\sum_{j=1}^{M} Y_{j} \omega_{\lambda X Y+}\left(\mathcal{J}_{j}\right) .
$$

By the general results of [Ru2, JP2] (see also [TM, FMU, JOP2]), $\operatorname{Ep}\left(\omega_{\lambda X Y+}\right) \geq 0$. The strict positivity of the entropy production for locally interacting fermionic reservoirs can be established by using either perturbative arguments (see [FMU]) or stability arguments (see Section 4.3 in [JP3] and [JP4]). This point is discussed in more detail in the forthcoming review [JP5].

To study linear response of $\omega_{\lambda X Y+}$, in addition to (A1)-(A2) we need the following regularity assumption.

(A3) For all $j$ and $g \in \mathcal{D}_{0}, h_{j} g \in \mathcal{D}$.

Our final assumption concerns time-reversal invariance.

(A4) There exists a complex conjugation $c$ on $\mathfrak{h}$ which commutes with all $h_{j}$ and satisfies $c g=g$ for all $g \in \mathcal{D}_{0}$.

If (A4) holds, then the map $\Theta\left(a^{\#}(f)\right)=a^{\#}(c f)$ extends to an involutive skew $*$-automorphism of $\mathcal{O}$ such that $\Theta \circ \tau_{j}^{t}=\tau_{j}^{-t} \circ \Theta$ and $\Theta(V)=V$. This implies that $\Theta \circ \tau_{\lambda}^{t}=\tau_{\lambda}^{-t} \circ \Theta$ for all $\lambda$. Note also that

$$
\Theta\left(\Phi_{j}\right)=-\Phi_{j}, \quad \Theta\left(\mathcal{J}_{j}\right)=-\mathcal{J}_{j}
$$

We set

$$
\begin{aligned}
\mathbb{I}_{\epsilon} & =\left\{(X, Y) \in \mathbb{R}^{2 M}|| X_{j}|<\epsilon,| Y_{j} \mid<\epsilon\right\}, \\
D_{\epsilon} & =\left\{(X, Y) \in \mathbb{C}^{2 M}|| X_{j}|<\epsilon,| Y_{j} \mid<\epsilon\right\}, \\
R_{\Lambda, \delta} & =\{\lambda \in \mathbb{C}|| \operatorname{Re} \lambda|<\Lambda,| \operatorname{Im} \lambda \mid<\delta\} .
\end{aligned}
$$

In the sequel $\mathfrak{F}_{j}$ stands for either $\Phi_{j}$ or $\mathcal{J}_{j}$. Our first result is:

Theorem 1.4 Suppose that Assumptions (A1)-(A3) hold and let $0<\Lambda<\Lambda_{0}$. Then there exist $\epsilon>0$ and $\delta>0$ such that the maps

$$
(\lambda, X, Y) \mapsto \omega_{\lambda X Y+}\left(\mathfrak{F}_{j}\right),
$$

extend to analytic functions on the set $R_{\Lambda, \delta} \times D_{\epsilon}$. In particular, for any $|\lambda|<\Lambda_{0}$ there exists $\epsilon(\lambda)>0$ such that the maps

$$
(X, Y) \mapsto \omega_{\lambda X Y+}\left(\mathfrak{F}_{j}\right)
$$

extend to analytic functions on $D_{\epsilon(\lambda)}$.

The kinetic transport coefficients are defined by

$$
\begin{gathered}
L_{\lambda \mathrm{hh}}^{k j}=\left.\partial_{X_{j}} \omega_{\lambda X Y+}\left(\Phi_{k}\right)\right|_{X=Y=0}, \\
L_{\lambda \mathrm{hc}}^{k j}=\left.\partial_{Y_{j}} \omega_{\lambda X Y+}\left(\Phi_{k}\right)\right|_{X=Y=0}, \\
L_{\lambda \mathrm{ch}}^{k j}=\left.\partial_{X_{j}} \omega_{\lambda X Y+}\left(\mathcal{J}_{k}\right)\right|_{X=Y=0}, \\
L_{\lambda \mathrm{cc}}^{k j}=\left.\partial_{Y_{j}} \omega_{\lambda X Y+}\left(\mathcal{J}_{k}\right)\right|_{X=Y=0},
\end{gathered}
$$


where the indices $\mathrm{h} / \mathrm{c}$ stand for heat/charge. For $A, B \in \mathcal{O}_{\vartheta}$ we set

$$
\mathcal{L}_{\lambda}(A, B)=\lim _{t \rightarrow+\infty} \frac{1}{2} \int_{-t}^{t} \omega_{\lambda \mathrm{eq}}\left(\tau_{\lambda}^{s}(A) B\right) \mathrm{d} s,
$$

and

$$
\mathfrak{L}_{\lambda}(A, B)=\lim _{t \rightarrow+\infty} \frac{1}{\beta_{\text {eq }}} \int_{0}^{t} \mathrm{~d} s \int_{0}^{\beta_{\text {eq }}} \mathrm{d} u \omega_{\lambda \text { eq }}\left(\tau_{\lambda}^{s}(A) \tau_{\lambda}^{\mathrm{i} u}(B)\right),
$$

whenever the limits exist. Our main result is:

Theorem 1.5 Suppose that Assumptions (A1)-(A3) hold and that $|\lambda|<\Lambda_{0}$. Then $\mathfrak{L}_{\lambda}(A, B)$ is well-defined for $A, B \in\left\{\Phi_{1}, \cdots, \Phi_{M}, \mathcal{J}_{1}, \cdots, \mathcal{J}_{M}\right\}$ and

$$
\begin{aligned}
& L_{\lambda \mathrm{hh}}^{k j}=\mathfrak{L}_{\lambda}\left(\Phi_{k}, \Phi_{j}\right), \\
& L_{\lambda \mathrm{hc}}^{k j}=\mathfrak{L}_{\lambda}\left(\Phi_{k}, \mathcal{J}_{j}\right), \\
& L_{\lambda \mathrm{ch}}^{k j}=\mathfrak{L}_{\lambda}\left(\mathcal{J}_{k}, \Phi_{j}\right), \\
& L_{\lambda \mathrm{cc}}^{k j}=\mathfrak{L}_{\lambda}\left(\mathcal{J}_{k}, \mathcal{J}_{j}\right) .
\end{aligned}
$$

Assume in addition that (A4) holds. Then $\mathcal{L}_{\lambda}(A, B)$ is well-defined for $A, B \in\left\{\Phi_{1} \cdots \Phi_{M}, \mathcal{J}_{1}, \cdots \mathcal{J}_{M}\right\}$,

$$
\begin{aligned}
& L_{\lambda \mathrm{hh}}^{k j}=\mathcal{L}_{\lambda}\left(\Phi_{k}, \Phi_{j}\right), \\
& L_{\lambda \mathrm{hc}}^{k j}=\mathcal{L}_{\lambda}\left(\Phi_{k}, \mathcal{J}_{j}\right), \\
& L_{\lambda \mathrm{ch}}^{k j}=\mathcal{L}_{\lambda}\left(\mathcal{J}_{k}, \Phi_{j}\right), \\
& L_{\lambda \mathrm{cc}}^{k j}=\mathcal{L}_{\lambda}\left(\mathcal{J}_{k}, \mathcal{J}_{j}\right),
\end{aligned}
$$

and

$$
\begin{aligned}
& L_{\lambda \mathrm{hh}}^{k j}=L_{\lambda \mathrm{hh}}^{j k}, \\
& L_{\lambda \mathrm{cc}}^{k j}=L_{\lambda \mathrm{cc}}^{j k}, \\
& L_{\lambda \mathrm{hc}}^{k j}=L_{\lambda \mathrm{ch}}^{j k} .
\end{aligned}
$$

Remark 1.The formulas (1.9) are the GKF without time reversal assumption. The formulas (1.10) are the GKF in the standard form. The formulas (1.11) are the Onsager reciprocity relations. The ORR are an immediate consequence of (1.10) and the KMS condition, see [JOP1, JOP2].

Remark 2. If $\bar{n}=1$, then our proofs give that Theorems 1.1-1.5 hold with $\Lambda_{0}=1 / 2 K \ell_{0}$. However, since in this case the coupled system is quasi-free, these theorems can be also proven using trace class scattering theory which yields better constants and wealth of additional information about the model. For more information about this special case we refer the reader to [AJPP1, AJPP2, JKP].

Remark 3. With regard to the Green-Kubo formulas (1.10), a natural question is whether the correlation functions $t \mapsto \omega_{\lambda \text { eq }}\left(\tau_{\lambda}^{t}(A) B\right)$ are absolutely integrable for $A, B \in\left\{\Phi_{1}, \cdots, \Phi_{M}, \mathcal{J}_{1}, \cdots \mathcal{J}_{M}\right\}$. This is a delicate dynamical problem which is studied in [JPP]. In this paper we only establish the existence of the improper integrals

$$
\lim _{t \rightarrow+\infty} \int_{-t}^{t} \omega_{\lambda \mathrm{eq}}\left(\tau_{\lambda}^{s}(A) B\right) \mathrm{d} s .
$$


Remark 4. By Theorem 1.4, the functions $\lambda \mapsto L_{\lambda \mathrm{uv}}^{k j}, \mathrm{u}, \mathrm{v} \in\{\mathrm{h}, \mathrm{c}\}$, are analytic for $|\lambda|<\Lambda_{0}$ and can be expanded into power series whose coefficients can be computed. Such computations can be used to verify that in specific examples the transport coefficients are non-vanishing. For reasons of space we shall discuss these perturbative computations in the forthcoming review [JP5].

Remark 5. Our results are tailored for application to tight-binding type models of electronic transport in which Assumption (A2) is usually satisfied. However, all our proofs extend to unbounded $h_{j}$ 's as long as $\mathcal{D}_{0} \subset \operatorname{Dom}\left(\mathrm{e}^{a\left|h_{j}\right|}\right)$ for all $j$ and some $a>\beta_{\text {eq. }}$. It is an interesting technical problem to prove Theorems 1.4 and 1.5 for unbounded $h_{j}$ 's without this additional technical assumption.

Remark 6. Theorems 1.1-1.3 are fairly flexible and are easily adapted to a number of different setups involving free Fermi gas reservoirs. The same applies to Theorems 1.4 and 1.5. For example, one may consider the tensor product structure, where the joint system in absence of interaction is described by $\mathcal{O}=\mathcal{O}_{1} \otimes \cdots \otimes \mathcal{O}_{M}$, $\tau_{0}=\tau_{1} \otimes \cdots \otimes \tau_{M}, \omega=\omega_{1} \otimes \cdots \otimes \omega_{M}$. This type of models was studied in [FMU]. Another class of related models are local perturbations of the exactly solvable Electronic Black-Box Model studied in [AJPP1, AJPP2]. Instead of coupled free fermionic systems one may consider coupled X - Y quantum spin chains. Theorems 1.4 and 1.5 extend to these models with only notational changes, see [JP5] for details.

Remark 7. We call $A \in \mathcal{O}$ centered if $\omega_{\lambda X Y}(A)=0$ for all $|\lambda|<\Lambda_{0}$ and $(X, Y) \in \mathbb{I}_{\epsilon}$. Our proof easily extends to the general Green-Kubo formulas

$$
\left.\partial_{X_{j}} \omega_{\lambda X Y+}(A)\right|_{X=Y=0}=\mathcal{L}_{\lambda}\left(A, \Phi_{j}\right),\left.\quad \partial_{Y_{j}} \omega_{\lambda X Y+}(A)\right|_{X=Y=0}=\mathcal{L}_{\lambda}\left(A, \mathcal{J}_{j}\right),
$$

for centered observables $A$ which are polynomials in $a^{\#}(f)$ with $f \in \mathcal{D}$.

We finish this subsection with some examples to which Theorems 1.1-1.5 apply. Let $\mathcal{G}$ be the set of vertices of a connected graph of bounded degree and $\Delta_{\mathcal{G}}$ the associated discrete Laplacian acting on $l^{2}(\mathcal{G})$. We recall that

$$
\left(\Delta_{\mathcal{G}} \psi\right)(x)=\sum_{|y-x|=1} \psi(y),
$$

where $|y-x|$ is the distance on the graph. $\Delta_{\mathcal{G}}$ is a bounded self-adjoint operator and $\left\|\Delta_{\mathcal{G}}\right\|=\sup _{x \in \mathcal{G}} d(x)$, where $d(x)$ is the degree of the vertex $x$. Let $\delta_{x}$ be the Kronecker delta function at $x \in \mathcal{G}$. We shall call the graph $\mathcal{G}$ admissible if there exists $\gamma>1$ such that for all $x, y \in \mathcal{G}$,

$$
\left|\left(\delta_{x}, \mathrm{e}^{-\mathrm{i} t \Delta_{\mathcal{G}}} \delta_{y}\right)\right|=O\left(|t|^{-\gamma}\right),
$$

as $t \rightarrow \infty$. Clearly, the discrete Laplacian of an admissible graph has purely absolutely continuous spectrum.

An example of admissible graph is $\mathcal{G}=\mathbb{Z}^{d}$ for $d \geq 3$. In this case $\gamma=d / 2$. Another example is the half-space $\mathcal{G}=\mathbb{Z}_{+} \times \mathbb{Z}^{d-1}$ where $\mathbb{Z}_{+}=\{0,1, \cdots\}$ and $d \geq 1$ (if $d=1$ then $\mathcal{G}=\mathbb{Z}_{+}$). In this case $\gamma=(d+2) / 2$. Tubular graphs of the type $\mathbb{Z}_{+} \times \Gamma$, where $\Gamma \subset \mathbb{Z}^{d-1}$ is finite, are admissible with $\gamma=3 / 2$. Another well-known admissible graph is a rooted Bethe lattice where $\gamma=3 / 2$.

Assumptions (A1)-(A4) and Theorems 1.1-1.5 hold if

(i) $\mathcal{G}_{1}, \ldots, \mathcal{G}_{M}$ are admissible graphs;

(ii) $\mathfrak{h}_{j}=\ell^{2}\left(\mathcal{G}_{j}\right)$ or more generally $\ell^{2}\left(\mathcal{G}_{j}\right) \otimes \mathbb{C}^{L}$ to allow for internal degrees of freedom (e.g., spin);

(iii) $\mathcal{D}$ is the subspace of finitely supported elements of $\mathfrak{h}$;

(iv) $h_{j}=-\Delta_{\mathcal{G}_{j}}$;

(v) $u_{k j}, v_{k j}$ belong to $\mathcal{D}$.

Allowed interactions include $V=V^{\text {hop }}+V^{\text {int }}$ where 
(i) $V^{\text {hop }}$ describes tunneling junctions between the reservoirs:

$$
V^{\mathrm{hop}}=\sum_{x, y} t(x, y)\left(a^{*}\left(\delta_{x}\right) a\left(\delta_{y}\right)+a^{*}\left(\delta_{y}\right) a\left(\delta_{x}\right)\right),
$$

where $t: \mathcal{G} \times \mathcal{G} \rightarrow \mathbb{R}$ is a finitely supported function $\left(\mathcal{G}=\cup_{j} \mathcal{G}_{j}\right)$;

(ii) $V^{\text {int }}$ is a local pair interaction

$$
V^{\mathrm{int}}=\sum_{x, y} v(x, y) a^{*}\left(\delta_{x}\right) a^{*}\left(\delta_{y}\right) a\left(\delta_{y}\right) a\left(\delta_{x}\right)
$$

where $v: \mathcal{G} \times \mathcal{G} \rightarrow \mathbb{R}$ is finitely supported.

This concrete model is studied in detail in [JP5].

\section{Basic properties of the model}

In this section we prove Theorems 1.1, 1.2, and 1.3.

\subsection{Proof of Theorem 1.1}

We start with some preliminaries which are of independent interest. Let $A=a_{1} \cdots a_{m}$ and $B=b_{1} \cdots b_{q}$ where the $a_{k}$ and $b_{j}$ are creation/annihilation operators. Thus, $A$ and $B$ are monomials of order $m$ and $q$ respectively. If $q$ is even it follows from the CAR that

$$
\begin{aligned}
{\left[B, a_{j}\right] } & =b_{1} \cdots b_{q} a_{j}-a_{j} b_{1} \cdots b_{q} \\
& =b_{1} \cdots b_{q} a_{j}-\left(\left\{b_{1}, a_{j}\right\}-b_{1} a_{j}\right) b_{2} \cdots b_{q} \\
& =-\left\{b_{1}, a_{j}\right\} b_{2} \cdots b_{q}+b_{1}\left(b_{2} \cdots b_{q} a_{j}+a_{j} b_{2} \cdots b_{q}\right) \\
& \vdots \\
& =\sum_{k=1}^{q}(-1)^{k}\left\{b_{k}, a_{j}\right\} b_{1} \cdots b_{k-1} b_{k+1} \cdots b_{q}
\end{aligned}
$$

and hence

$$
\begin{aligned}
{[B, A] } & =\sum_{j=1}^{m} a_{1} \cdots a_{j-1}\left[B, a_{j}\right] a_{j+1} \cdots a_{m} \\
& =\sum_{j=1}^{m} \sum_{k=1}^{q}(-1)^{k}\left\{b_{k}, a_{j}\right\} a_{1} \cdots a_{j-1} b_{1} \cdots b_{k-1} b_{k+1} \cdots b_{n} a_{j+1} \cdots a_{m} .
\end{aligned}
$$

The anticommutator $\left\{b_{k}, a_{j}\right\}$ on the right hand side is called contraction of the factor $b_{k}$ of $B$ with the factor $a_{j}$ of $A$. Note that contractions are numbers.

Iterating the last formula we get, for any monomials $B_{1}, B_{2}, \ldots B_{n+1}$ of even orders $q_{1}, q_{2}, \ldots q_{n+1}$ and any monomial $A$ of order $m$

$$
\left[B_{n+1},\left[\tau_{0}^{s_{n}}\left(B_{n}\right),\left[\cdots,\left[\tau_{0}^{s_{1}}\left(B_{1}\right), \tau_{0}^{s_{0}}(A)\right] \cdots\right]\right]\right]=\sum_{p \in \mathcal{P}_{n}\left(A, B_{1}, \ldots, B_{n+1}\right)} G_{p}\left(s_{0}, \cdots, s_{n}\right) F_{p}
$$

where the $F_{p}$ are monomials of order $q_{1}+q_{2}+\cdots+q_{n+1}+m-2(n+1)$ and the coefficients $G_{p}$ are products of $n+1$ contractions. The sum on the right hand side runs over the set $\mathcal{P}_{n}\left(A, B_{1}, \ldots, B_{n+1}\right)$ whose elements $p$ are contraction diagrams of the type displayed in Figure 1. 


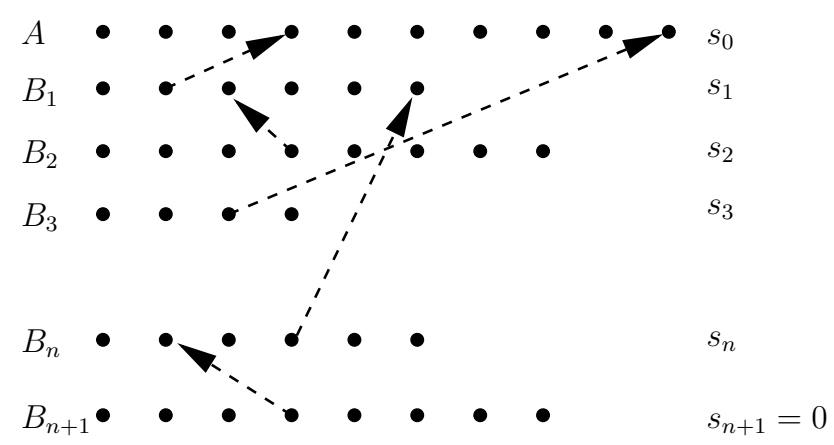

Figure 1: An element of the set $\mathcal{P}_{n}$.

Each line of this diagram represents a monomial, as labeled on the left. Each dot on a line represents a factor of the corresponding monomial. The dashed lines represent contractions of such factors. From each line of the diagram there is exactly one contraction going up and any factor can belong only to one contraction. To a contraction diagram $p$ we associate its skeleton: a rooted tree $T$ whose nodes are 0 (the root), $1, \ldots, n+1$ and whose bonds correspond to the contractions (see Figure 2). The skeleton $T$ is simply obtained by collapsing each line of the contraction diagram $p$ to a single node. If there is an arrow going from the node $j$ to the node $k$ in $T$ we say that $j$ is a child of $k$ or that $k$ is the parent of $j$ (each node has a unique parent and we shall say that the root node 0 is its own parent). We can describe the rooted tree $T$ by the function $T:\{0, \ldots, n+1\} \rightarrow\{0, \ldots, n+1\}$ which to a node $j$ associates its parent $T(j)$. Reciprocally, any function $T$ such that $T(0)=0$ and $T(j)<j$ for $j=1, \ldots, n+1$ defines a rooted tree $T$. Such a function is called a climber of order $n+1$ and there is a one-to-one correspondence between climbers and rooted trees.

Suppose that all the factors of the monomials $B_{1}, \ldots, B_{n+1}$ are from $\left\{a^{\#}(g) \mid g \in \mathcal{D}_{0}\right\}$ and let $A$ and $\mathcal{A}$ be as in Theorem 1.1. Then, the factors of the monomials $F_{p}$ are from

$$
\left\{a^{\#}\left(\mathrm{e}^{\mathrm{i} s h_{0}} g\right) \mid g \in \mathcal{D}_{0}, s \in\left\{0, s_{1}, \ldots, s_{n}\right\}\right\} \cup\left\{a^{\#}\left(\mathrm{e}^{\mathrm{i} s_{0} h_{0}} g\right) \mid g \in \mathcal{A}\right\}
$$

The number of factors from the first set does not exceed $(n+1)(\bar{q}-1)$, where $\bar{q}=\max q_{k}$. The number of factors from the second set does not exceed $m-1$. If we denote

$$
S_{k}(t) \equiv \begin{cases}\sup _{f \in \mathcal{D}_{0}, g \in \mathcal{A}}\left|\left(f, \mathrm{e}^{\mathrm{i} t h_{0}} g\right)\right| & \text { for } k=0, \\ \sup _{f, g \in \mathcal{D}_{0}}\left|\left(f, \mathrm{e}^{\mathrm{i} t h_{0}} g\right)\right| & \text { for } k>0,\end{cases}
$$

then all coefficients $G_{p}$ associated with a given skeleton tree $T$ are bounded by

$$
\left|G_{p}\right| \leqslant \prod_{j=1}^{n+1} S_{T(j)}\left(s_{T(j)}-s_{j}\right) \equiv S(T)
$$

where we set $s_{n+1}=0$. Thus, if $N(T)$ denotes the number of contraction diagrams with skeleton tree $T$ we have

$$
\sum_{p \in \mathcal{P}_{n}}\left|G_{p}\right| \leqslant \sum_{T \in \mathcal{T}_{n+1}} N(T) S(T),
$$

where $\mathcal{T}_{n+1}$ denotes the set of all rooted trees with nodes 0 (the root), $1, \ldots, n+1$. Let us compute $N(T)$. To this end denote by $r_{j}$ the number of childs of the node $j$. For the tree of Figure 2 we have for example $r_{0}=2, r_{1}=2$, $r_{2}=r_{3}=0, r_{n}=1$ and $r_{n+1}=0$. Clearly, $N(T)=0$ if $r_{0}>m$ or $r_{j}>q_{j}-1$. Otherwise, to construct a 


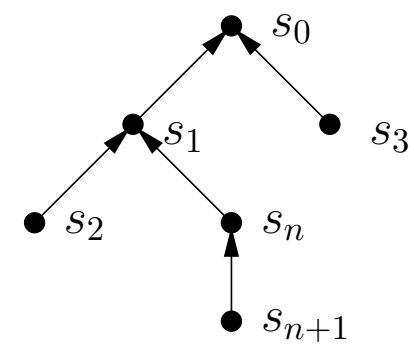

Figure 2: The skeleton tree corresponding to Figure 1.

diagram $p$ whose skeleton is $T$ we first have to choose a factor on each line $B_{1}, \ldots, B_{n+1}$. The number of such choices is clearly $q_{1} q_{2} \cdots q_{n+1}$. Now on line $A$ we have to choose one factor for each of the $r_{0}$ childs of node 0 . There are $m(m-1) \cdots\left(m-r_{0}+1\right)$ such choices. Similarly, on line $B_{1}$ we have to chose $r_{1}$ factors out of the $q_{1}-1$ remaining. There are $\left(q_{1}-1\right)\left(q_{1}-2\right) \cdots\left(q_{1}-r_{1}\right)$ such choices. The same reasoning applies to lines $B_{2}, \ldots, B_{n}$, and we conclude that

$$
N(T)=\frac{m !}{\left(m-r_{0}\right) !} \prod_{j=1}^{n} \frac{q_{j} !}{\left(q_{j}-r_{j}-1\right) !} \leq \frac{m !}{\left(m-r_{0}\right) !} \prod_{j=1}^{n} \frac{\bar{q} !}{\left(\bar{q}-r_{j}-1\right) !} \equiv \bar{N}(T) .
$$

We now turn to the proof of Theorem 1.1. Since $V=\sum_{k=1}^{K} V_{k}$ where $V_{k}$ are monomials of order $q_{k}=2 n_{k}$ (so $\bar{q}=2 \bar{n})$, we can write

$$
\mathcal{C}_{A}^{(n)}\left(s_{0}, \ldots, s_{n}\right)=\sum_{k_{1}, \ldots, k_{n+1}=1}^{K}\left[V_{k_{n+1}},\left[\tau_{0}^{s_{n}}\left(V_{k_{n}}\right),\left[\cdots,\left[\tau_{0}^{s_{1}}\left(V_{k_{1}}\right), \tau_{0}^{s_{0}}(A)\right] \cdots\right]\right]\right]
$$

and Parts (1) and (2) follow immediately with

$$
\mathcal{P}_{n}(A) \equiv \bigcup_{k_{1}, \ldots, k_{n+1}=1}^{K}\left\{\left(k_{1}, \ldots, k_{n+1}\right)\right\} \times \mathcal{P}_{n}\left(A, V_{k_{1}}, \ldots, V_{k_{n+1}}\right)
$$

To prove (3), we start with the estimate

$$
\sum_{p \in \mathcal{P}_{n}(A)}\left|G_{A, p}^{(n)}\right| \leq K^{n+1} \sum_{T \in \mathcal{T}_{n+1}} \bar{N}(T) S(T) .
$$

Hence,

$$
W \equiv \sum_{n=0}^{\infty}|\lambda|^{n+1} \sum_{p \in \mathcal{P}_{n}(A)} \int_{0 \leq s_{n} \leq \cdots \leq s_{0}}\left|G_{A, p}^{(n)}\left(s_{0}, \ldots, s_{n}\right)\right| \mathrm{d} s_{0} \cdots \mathrm{d} s_{n}
$$

satisfies

$$
W \leq W_{0} \equiv \sum_{n=1}^{\infty} \sum_{T \in \mathcal{T}_{n}} \bar{N}(T) \int_{0=s_{n} \leq s_{n-1} \cdots \leq s_{0}} \prod_{j=1}^{n}\left(|\lambda| K S_{T(j)}\left(s_{T(j)}-s_{j}\right)\right) \mathrm{d} s_{0} \cdots \mathrm{d} s_{n-1}
$$

We will need the following general result of [BGM]. 
Theorem 2.1 Let $m_{k}, \widetilde{m}_{k}$ be two sequences of nonnegative numbers and $g, \widetilde{g}$ two integrable nonnegative functions on $\left[0, \infty\left[\right.\right.$. Denote by $\|g\|$ and $\|\widetilde{g}\|$ their $L^{1}$-norms, set $g_{0}=\widetilde{g}$ and $g_{k}=g$ for $k>0$ and define

$$
M(x) \equiv \sum_{k=0}^{\infty} \frac{m_{k}}{k !} x^{k}, \quad \widetilde{M}(x) \equiv \sum_{k=0}^{\infty} \frac{\widetilde{m}_{k}}{k !} x^{k} .
$$

To any rooted tree $T \in \mathcal{T}_{n}$ associate the weight (recall that $r_{j}$ is the number of childs of the node $j$ ),

$$
w(T)=\widetilde{m}_{r_{0}} m_{r_{1}} \cdots m_{r_{n}} \int_{0=s_{n} \leq s_{n-1} \leq \cdots \leq s_{0}} \prod_{j=1}^{n} g_{T(j)}\left(s_{T(j)}-s_{j}\right) \mathrm{d} s_{0} \cdots \mathrm{d} s_{n-1} .
$$

Then, the sum $W=\sum_{n=1}^{\infty} \sum_{T \in \mathcal{T}_{n}} w(T)$ is finite if and only if the equation $M(\|g\| x)=x$ has a positive solution $x$ such that $\widetilde{M}(\|\widetilde{g}\| x)<\infty$. If $x^{*}$ denotes the least such solution, then $W=\widetilde{M}\left(\|\widetilde{g}\| x^{*}\right)$.

To apply this result we set $\widetilde{m}_{k}=0$ for $k=0$ and $k>m$, otherwise

$$
\tilde{m}_{k}=\frac{m !}{(m-k) !}
$$

$m_{k}=0$ for $k \geq 2 \bar{n}$, otherwise

$$
m_{k}=\frac{(2 \bar{n}) !}{(2 \bar{n}-k-1) !}
$$

and

$$
g(s)=|\lambda| K S_{1}(s), \quad \widetilde{g}(s)=|\lambda| K S_{0}(s) .
$$

Hence, $M(x)=2 \bar{n}(1+x)^{2 \bar{n}-1}, \widetilde{M}(x)=(1+x)^{m}-1,\|g\|=|\lambda| K \ell_{0}$, and $\|\widetilde{g}\|=|\lambda| K \ell$. An elementary analysis shows that, if

$$
\Lambda_{0}= \begin{cases}\frac{1}{2 K \ell_{0}} & \text { for } \bar{n}=1, \\ \frac{1}{2 \bar{n} K \ell_{0}} \frac{(2 \bar{n}-2)^{2 \bar{n}-2}}{(2 \bar{n}-1)^{2 \bar{n}-1}} & \text { for } \bar{n}>1,\end{cases}
$$

then, as long as $|\lambda|<\Lambda_{0}$ for $\bar{n}=1$ and $|\lambda| \leq \Lambda_{0}$ for $\bar{n}>1$, the equation $M(\|g\| x)=x$ has a least positive solution $x^{*}$ satisfying

$$
0 \leq x^{*} \leq \frac{2 \bar{n}}{\left(1-|\lambda| / \Lambda_{0}\right)+2 \bar{n}(2 \bar{n}-2) K \ell_{0}|\lambda|}
$$

and that

$$
W \leq W_{0}=\left(1+K \ell|\lambda| x^{*}\right)^{m}-1 \leq\left(1+\frac{2 \bar{n} K \ell|\lambda|}{\left(1-|\lambda| / \Lambda_{0}\right)+2 \bar{n}(2 \bar{n}-2) K \ell_{0}|\lambda|}\right)^{m}-1
$$

This ends the proof of Theorem 1.1.

\subsection{Proofs of Theorems 1.2 and 1.3}

Proof of Theorem 1.2. To establish the existence of the limit (1.6) for all $A \in \mathcal{O}$ it suffices to consider the case $A=a^{\#}(f)$ with $f \in \mathcal{D}$ and $\|f\|=1$. Since

$$
\tau_{0}^{-t_{2}} \circ \tau_{\lambda}^{t_{2}}(A)-\tau_{0}^{-t_{1}} \circ \tau_{\lambda}^{t_{1}}(A)=\mathrm{i} \lambda \int_{t_{1}}^{t_{2}} \tau_{0}^{-s}\left(\left[V, \tau_{\lambda}^{s}(A)\right]\right) \mathrm{d} s,
$$


we have that

$$
\left\|\tau_{0}^{-t_{2}} \circ \tau_{\lambda}^{t_{2}}(A)-\tau_{0}^{-t_{1}} \circ \tau_{\lambda}^{t_{1}}(A)\right\| \leq|\lambda| \int_{t_{1}}^{t_{2}}\left\|\left[V, \tau_{\lambda}^{s}(A)\right]\right\| \mathrm{d} s .
$$

The expansion (1.1) yields

$$
\left[V, \tau_{\lambda}^{s}(A)\right]=\left[V, \tau_{0}^{s}(A)\right]+\sum_{n=1}^{\infty}(\mathrm{i} \lambda)^{n} \int_{0 \leq s_{n} \leq \cdots \leq s_{1} \leq s}\left[V,\left[\tau_{0}^{s_{n}}(V),\left[\cdots,\left[\tau_{0}^{s_{1}}(V), \tau_{0}^{s}(A)\right] \cdots\right]\right]\right] \mathrm{d} s_{1} \cdots \mathrm{d} s_{n} .
$$

Our standing assumption (1.3) and the fact that $\|f\|=1$ implies that $\left\|F_{A, p}^{(n)}\right\| \leq 1$ and we can estimate

$$
\left\|\left[V,\left[\tau_{0}^{s_{n}}(V),\left[\cdots,\left[\tau_{0}^{s_{1}}(V), \tau_{0}^{s}(A)\right] \cdots\right]\right]\right]\right\| \leq \sum_{p \in \mathcal{P}_{n}(A)}\left|G_{A, p}^{(n)}\left(s, s_{1}, \cdots, s_{n}\right)\right| .
$$

Part (3) of Theorem 1.1 yields that for $|\lambda|<\Lambda_{0}$,

$$
\int_{0}^{\infty}\left\|\left[V, \tau_{\lambda}^{s}(A)\right]\right\| \mathrm{d} s<\infty
$$

The estimates (2.14) and (2.15) imply the existence of the limit (1.6) for $|\lambda|<\Lambda_{0}$.

The map $\gamma_{\lambda}^{+}$is obviously a $*$-morphism. To prove that it is an isomorphism, it suffices to show that the limits

$$
\lim _{t \rightarrow+\infty} \tau_{\lambda}^{-t} \circ \tau_{0}^{t}(A)
$$

exist for all $A \in \mathcal{O}$. Repeating the above argument we see that it suffices to show that

$$
\int_{0}^{\infty}\left\|\left[V, \tau_{0}^{s}(A)\right]\right\| \mathrm{d} s<\infty
$$

for $A=a^{\#}(f), f \in \mathcal{D}$. But this is a special case of Equ. (2.15).

Proof of Theorem 1.3. Since $h$ has purely absolutely continuous spectrum the quantum dynamical system $\left(\mathcal{O}, \tau_{0}, \omega\right)$ has the property of return to equilibrium: for all $\omega$-normal states $\eta$ and $A \in \mathcal{O}$,

$$
\lim _{|t| \rightarrow \infty} \eta \circ \tau_{0}^{t}(A)=\omega(A)
$$

see, e.g., [AJPP1]. The existence of norm-limits (1.6) ensures that

$$
\lim _{t \rightarrow+\infty} \eta\left(\tau_{\lambda}^{t}(A)\right)=\lim _{t \rightarrow+\infty} \eta \circ \tau_{0}^{t}\left(\tau_{0}^{-t} \circ \tau_{\lambda}^{t}(A)\right)=\lim _{t \rightarrow+\infty} \eta \circ \tau_{0}^{t}\left(\gamma_{\lambda}^{+}(A)\right)=\omega\left(\gamma_{\lambda}^{+}(A)\right),
$$

and the statement follows.

\section{Proofs of Theorems 1.4 and 1.5}

\subsection{Strategy}

The strategy of the proofs of Theorems 1.4 and 1.5 is based on the arguments in [JOP3]. Consider the $C^{*}$-dynamics $\sigma_{X Y}$ on $\mathcal{O}$ generated by

$$
\delta_{X Y}=\delta_{0}-\mu_{\mathrm{eq}} \xi-\sum_{j} \frac{X_{j}}{\beta_{\mathrm{eq}}} \delta_{j}-\sum_{j} \frac{Y_{j}}{\beta_{\mathrm{eq}}} \xi_{j}
$$


The reference state $\omega_{X Y}$ is the unique $\left(\sigma_{X Y}, \beta_{\text {eq }}\right)$-KMS state on $\mathcal{O}$. Let $\sigma_{\lambda X Y}$ be the $C^{*}$-dynamics on $\mathcal{O}$ generated by

$$
\delta_{\lambda X Y}=\delta_{X Y}+\mathrm{i} \lambda[V, \cdot]
$$

The Araki perturbation theory [Ar, BR2, DJP] yields that there exists a unique $\left(\sigma_{\lambda X Y}, \beta_{\text {eq }}\right)$-KMS state on $\mathcal{O}$. We denote this state by $\omega_{\lambda X Y}$. The states $\omega_{X Y}$ and $\omega_{\lambda X Y}$ are mutually normal.

Recall that $\mathfrak{F}_{j}$ stands for either $\Phi_{j}$ or $\mathcal{J}_{j}$. Our main technical result is:

Theorem 3.1 Suppose that Assumptions (A1)-(A3) hold and let $0<\Lambda<\Lambda_{0}$. Then there exist $\epsilon>0$ and $\delta>0$ such that for all $t \geq 0$ the functions $(\lambda, X, Y) \mapsto \omega_{\lambda X Y}\left(\tau_{\lambda}^{t}\left(\mathfrak{F}_{j}\right)\right)$ have analytic extensions to $R_{\Lambda, \delta} \times D_{\epsilon}$ satisfying

$$
\sup _{\lambda \in R_{\Lambda, \delta},(X, Y) \in D_{\epsilon}, t \geq 0}\left|\omega_{\lambda X Y}\left(\tau_{\lambda}^{t}\left(\mathfrak{F}_{j}\right)\right)\right|<\infty .
$$

This result and the multi-variable Vitali theorem yield Theorem 1.4 (see Theorem 2.3 in [JOP3]). Moreover, the relations

$$
\partial_{X_{j}} \omega_{\lambda X Y+}\left(\mathfrak{F}_{k}\right)=\lim _{t \rightarrow+\infty} \partial_{X_{j}} \omega_{\lambda X Y} \circ \tau_{\lambda}^{t}\left(\mathfrak{F}_{k}\right), \quad \partial_{Y_{j}} \omega_{\lambda X Y+}\left(\mathfrak{F}_{k}\right)=\lim _{t \rightarrow+\infty} \partial_{Y_{j}} \omega_{\lambda X Y} \circ \tau_{\lambda}^{t}\left(\mathfrak{F}_{k}\right)
$$

hold for $(\lambda, X, Y) \in R_{\Lambda, \delta} \times D_{\epsilon}$. The proof of Relations (1.9) is completed by invoking the following identities proven in [JOP1, JOP2]:

$$
\begin{gathered}
\left.\partial_{X_{j}} \omega_{\lambda X Y}\left(\tau_{\lambda}^{t}\left(\mathfrak{F}_{k}\right)\right)\right|_{X=Y=0}=\frac{1}{\beta_{\mathrm{eq}}} \int_{0}^{t} \mathrm{~d} s \int_{0}^{\beta_{\mathrm{eq}}} \mathrm{d} u \omega_{\lambda \mathrm{eq}}\left(\tau_{\lambda}^{s}\left(\mathfrak{F}_{k}\right) \tau_{\lambda}^{\mathrm{i} u}\left(\Phi_{j}\right)\right), \\
\left.\partial_{Y_{j}} \omega_{\lambda X Y}\left(\tau_{\lambda}^{t}\left(\mathfrak{F}_{k}\right)\right)\right|_{X=Y=0}=\frac{1}{\beta_{\mathrm{eq}}} \int_{0}^{t} \mathrm{~d} s \int_{0}^{\beta_{\mathrm{eq}}} \mathrm{d} u \omega_{\lambda \mathrm{eq}}\left(\tau_{\lambda}^{s}\left(\mathfrak{F}_{k}\right) \tau_{\lambda}^{\mathrm{i} u}\left(\mathcal{J}_{j}\right)\right) .
\end{gathered}
$$

Proposition 4.1 in [JOP2] yields that (1.9) and time-reversal invariance (A4) imply (1.10). The KMS condition and (1.10) imply (1.11) [JOP1, JOP2]. Hence, to complete the proofs of Theorems 1.4 and 1.5 we need to establish Theorem 3.1.

\subsection{Proof of Theorem 3.1}

The GNS representation of the algebra $\mathcal{O}$ associated to the gauge-invariant quasi-free state $\omega_{X Y}$ can be explicitly computed [AW, BR2]. Let $\mathcal{F}$ be the anti-symmetric Fock space over $\mathfrak{h}$. We denote by $\Omega_{\mathrm{f}}$ the vacuum vector and by $N$ the number operator. Let

$$
\mathcal{H}=\mathcal{F} \otimes \mathcal{F}, \quad \Omega=\Omega_{\mathrm{f}} \otimes \Omega_{\mathrm{f}}
$$

In the sequel $\mathcal{B}(\mathfrak{H})$ denotes the $C^{*}$-algebra of all bounded operators on a Hilbert space $\mathfrak{H}$. Let $\mathcal{C}_{j}$ be given complex conjugations on $\mathfrak{h}_{j}$ and $\mathcal{C}=\oplus_{j} \mathcal{C}_{j}$. Without loss of generality we may assume that $\mathcal{C}_{j}$ commutes with $h_{j}$. As usual, we denote $\mathcal{C} f=\bar{f}$. The map

$$
\pi_{X Y}(a(f))=a\left(\left(I-T_{X Y}\right)^{1 / 2} f\right) \otimes I+(-I)^{N} \otimes a^{*}\left(T_{X Y}^{1 / 2} \bar{f}\right)
$$

uniquely extends to a representation $\pi_{X Y}: \mathcal{O} \rightarrow \mathcal{B}(\mathcal{H})$ and the triple $\left(\mathcal{H}, \pi_{X Y}, \Omega\right)$ is the GNS-representation of the algebra $\mathcal{O}$ associated to the state $\omega_{X Y}$.

In what follows we suppose that Assumptions (A1)-(A3) hold. By adding a constant to $\mu_{\mathrm{eq}}$ without loss of generality we may assume that $h_{j} \geq 0$. 
Lemma 3.2 For $\beta>0$ and $\mu \in \mathbb{R}$ set

$$
\epsilon(\beta, \mu)=\frac{\pi \beta}{\pi+4 \beta(|\mu|+1)}<\beta
$$

The functions

$$
l_{ \pm}(s, x, y)=\left(1+\mathrm{e}^{ \pm[(\beta-x) s-(\beta \mu+y)]}\right)^{-1 / 2},
$$

are continuous and, for fixed s, analytic in $(x, y)$ on the set $\left\{(s, x, y) \in \mathbb{R}_{+} \times \mathbb{C}^{2}|| x|<\epsilon(\beta, \mu)| y \mid,<\epsilon(\beta, \mu)\right\}$. Moreover, for any $\delta<\epsilon(\beta, \mu)$ one has

$$
\sup _{s \in \mathbb{R}_{+},(x, y) \in \mathbb{C}^{2},|x|<\delta,|y|<\delta}\left|l_{ \pm}(s, x, y)\right|<\infty .
$$

Proof. Set $x=a+\mathrm{i} b$ and $y=c+\mathrm{i} d$ with $a, b, c, d \in \mathbb{R}, M_{\delta}=\left\{(x, y) \in \mathbb{C}^{2}|| x|<\delta| y \mid,<\delta\right\}$ and write the exponent in $l_{-}$as

$$
\theta(s, x, y)=-\left(u(s)\left(1-\mathrm{i} \frac{b}{\beta-a}\right)-\mathrm{i}\left(d+b \frac{\beta \mu+c}{\beta-a}\right)\right),
$$

where

$$
u(s)=(\beta-a)\left(s-\frac{\beta \mu+c}{\beta-a}\right) .
$$

If $(x, y) \in M_{\delta}$ with $\delta<\beta$, then

$$
\left|\frac{b}{\beta-a}\right|<\frac{\delta}{\beta-\delta}, \quad\left|d+b \frac{\beta \mu+c}{\beta-a}\right|<\delta \frac{\beta(1+|\mu|)}{\beta-\delta},
$$

and it follows that $\theta\left(\mathbb{R}_{+} \times M_{\delta}\right)$ is contained in the dashed region of Figure 3. An elementary calculation shows that for $\delta<\epsilon(\beta, \mu)$ this region does not intersect the half-lines $\mathbb{R}_{+} \pm \mathrm{i} \pi / 2$. Another elementary calculation shows that $1+\mathrm{e}^{\theta\left(\mathbb{R}_{+} \times M_{\delta}\right)}$ is contained in a bounded region of the half-plane

$$
\left\{z \in \mathbb{C} \mid \operatorname{Re} z>1-\mathrm{e}^{-\pi \beta\left(\delta^{-1}-\epsilon(\beta, \mu)^{-1}\right)}\right\} .
$$

Thus, $l_{-}$is a bounded continuous function on $\mathbb{R}_{+} \times M_{\delta}$ which is clearly analytic in $(x, y)$ for any fixed $s \in \mathbb{R}_{+}$. This yields the result since $\mathrm{e}^{\theta / 2}$ has obviously the same properties and $l_{+}=\mathrm{e}^{\theta / 2} l_{-}$for real $s, x, y$.

The spectral theorem and Lemma 3.2 yield

Lemma 3.3 The maps

$$
(X, Y) \mapsto\left(I-T_{X Y}\right)^{1 / 2} \in \mathcal{B}(\mathfrak{h}), \quad(X, Y) \mapsto T_{X Y}^{1 / 2} \in \mathcal{B}(\mathfrak{h}),
$$

extend to analytic $\mathcal{B}(\mathfrak{h})$-valued functions on $D_{\epsilon\left(\beta_{\mathrm{eq}}, \mu_{\mathrm{eq}}\right)}$.

Since for $X, Y$ real, $\left\|\pi_{X Y}\left(a^{\#}(f)\right)\right\|=\|f\|$, Lemma 3.3 implies

Lemma 3.4 For any $\delta>0$ there exists $\epsilon(\delta)>0$ such that for all $f \in \mathfrak{h}$ the operator-valued function

$$
(X, Y) \mapsto \pi_{X Y}\left(a^{\#}(f)\right) \in \mathcal{B}(\mathcal{H}),
$$

has an analytic extension to $D_{\epsilon(\delta)}$ which satisfies

$$
\sup _{(X, Y) \in D_{\epsilon(\delta)}}\left\|\pi_{X Y}\left(a^{\#}(f)\right)\right\| \leq(1+\delta)\|f\| .
$$




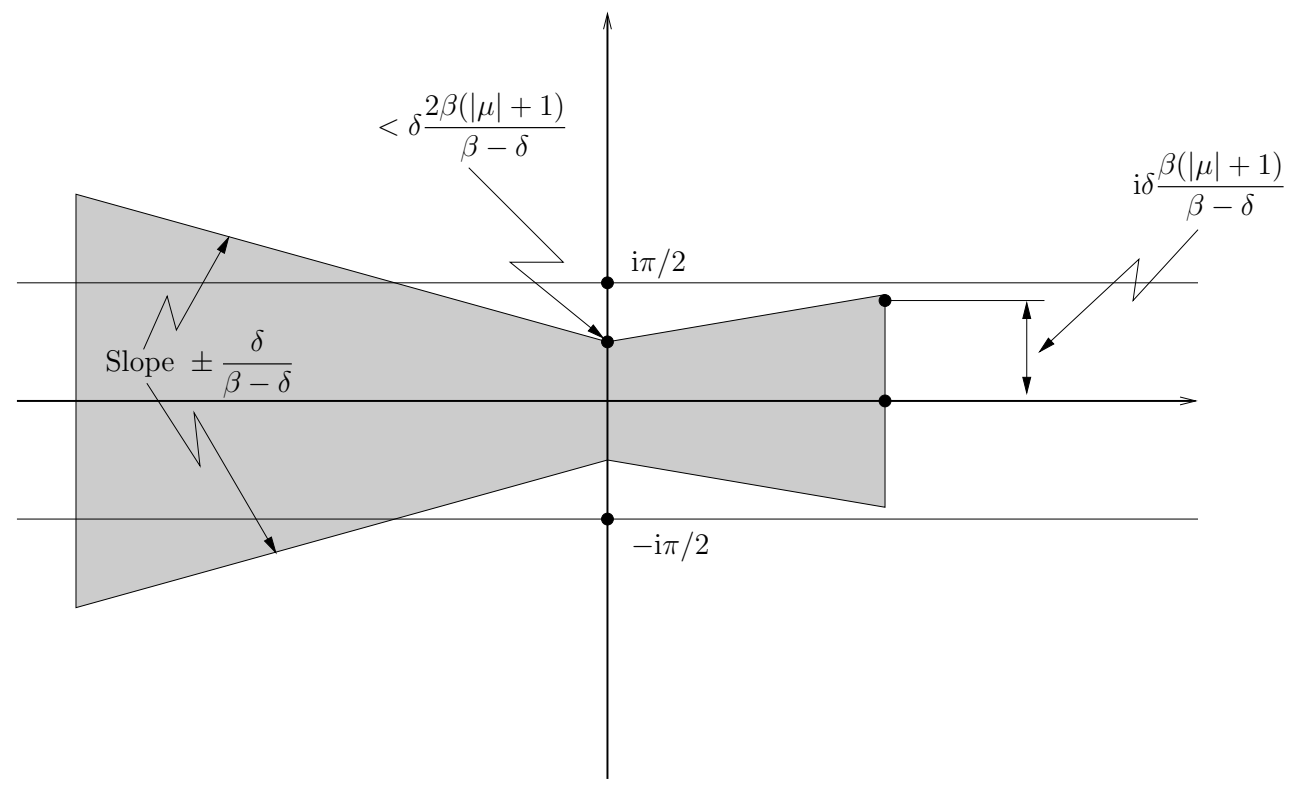

Figure 3: The range of the exponent $\theta(s, x, y)$.

Recall that $\delta_{X Y}$ is defined by (3.16). Let

$$
\begin{aligned}
h_{X Y} & =h_{0}-\mu_{\mathrm{eq}}-\sum_{j} \frac{X_{j}}{\beta_{\mathrm{eq}}} h_{j}-\sum_{j} \frac{Y_{j}}{\beta_{\mathrm{eq}}} p_{j} \\
& =\sum_{j}\left[\frac{\beta_{\mathrm{eq}}-X_{j}}{\beta_{\mathrm{eq}}} h_{j}-\frac{\beta_{\mathrm{eq}} \mu_{\mathrm{eq}}+Y_{j}}{\beta_{\mathrm{eq}}} p_{j}\right],
\end{aligned}
$$

where $p_{j}$ is the orthogonal projection on $\mathfrak{h}_{j}$. Clearly, $\mathrm{e}^{t \delta_{X Y}}\left(a^{\#}(f)\right)=a^{\#}\left(\mathrm{e}^{\mathrm{i} t h_{X Y}} f\right)$ is, for fixed $t$, an analytic function of $X, Y$.

Set

$$
V_{X Y}(s)=\sum_{k=1}^{K} \prod_{j=1}^{n_{k}} a^{*}\left(\mathrm{e}^{-s h_{X Y}} u_{k j}\right) a\left(\mathrm{e}^{s h_{X Y}} v_{k j}\right)
$$

and

$$
\mathcal{G}_{\lambda X Y}=\mathbb{1}+\sum_{n \geq 1}\left(-\lambda \beta_{\mathrm{eq}}\right)^{n} \int_{0 \leq s_{n} \leq \cdots \leq s_{1} \leq 1} V_{X Y}\left(\beta_{\mathrm{eq}} s_{n}\right) \cdots V_{X Y}\left(\beta_{\mathrm{eq}} s_{1}\right) \mathrm{d} s_{1} \cdots \mathrm{d} s_{n} .
$$

Araki's perturbation theory [Ar, BR2, DJP] yields that for $X, Y$ real the state $\omega_{\lambda X Y}$ can be expressed in terms of $\omega_{X Y}$ as

$$
\omega_{\lambda X Y}(A)=\frac{\omega_{X Y}\left(A \mathcal{G}_{\lambda X Y}\right)}{\omega_{X Y}\left(\mathcal{G}_{\lambda X Y}\right)}
$$

Lemma 3.5 The function

$$
(t, \lambda, X, Y) \mapsto \pi_{X Y}\left(\tau_{0}^{t}\left(\mathcal{G}_{\lambda X Y}\right)\right) \in \mathcal{B}(\mathcal{H}),
$$

extends to a continuous function on $\mathbb{R} \times \mathbb{C} \times D_{\epsilon\left(\beta_{\mathrm{eq}}, \mu_{\mathrm{eq}}\right)}$ which is analytic in $(\lambda, X, Y)$ for fixed $t$. Moreover, for all $\Lambda>0$ and $0<\epsilon<\epsilon\left(\beta_{\mathrm{eq}}, \mu_{\mathrm{eq}}\right)$,

$$
\sup _{t \in \mathbb{R}, \lambda \in \mathbb{C},|\lambda|<\Lambda,(X, Y) \in D_{\epsilon}}\left\|\pi_{X Y}\left(\tau_{0}^{t}\left(\mathcal{G}_{\lambda X Y}\right)\right)\right\|<\infty .
$$


Proof. Since for $X, Y$ real,

$\pi_{X Y}\left(\tau_{0}^{t}\left(\mathcal{G}_{\lambda X Y}\right)\right)=\mathbb{1}+\sum_{n \geq 1}\left(-\lambda \beta_{\mathrm{eq}}\right)^{n} \int_{0 \leq s_{n} \leq \cdots \leq s_{1} \leq 1} \pi_{X Y}\left(V_{X Y}\left(\beta_{\mathrm{eq}} s_{n}, t\right)\right) \cdots \pi_{X Y}\left(V_{X Y}\left(\beta_{\mathrm{eq}} s_{1}, t\right)\right) \mathrm{d} s_{1} \cdots \mathrm{d} s_{n}$

where

$$
\pi_{X Y}\left(V_{X Y}\left(\beta_{\mathrm{eq}} s, t\right)\right)=\sum_{k=1}^{K} \prod_{j=1}^{n_{k}} \pi_{X Y}\left(a^{*}\left(\mathrm{e}^{-\beta_{\mathrm{eq}} s h_{X Y}} \mathrm{e}^{\mathrm{i} t h_{0}} u_{k j}\right)\right) \pi_{X Y}\left(a\left(\mathrm{e}^{\beta_{\mathrm{eq}} s h_{X Y}} \mathrm{e}^{\mathrm{i} t h_{0}} v_{k j}\right)\right),
$$

the statement follows from Lemma 3.4.

Lemma 3.6 For all $t$ and $A \in \mathcal{O}$,

$$
\omega_{\lambda X Y}\left(\tau_{\lambda}^{t}(A)\right)=\omega_{\lambda X Y}\left(\tau_{0}^{t}(A)\right)+\frac{\mathrm{i} \lambda}{\omega_{X Y}\left(\mathcal{G}_{\lambda X Y}\right)} \int_{0}^{t} \omega_{X Y}\left(\left[V, \tau_{\lambda}^{s}(A)\right] \tau_{0}^{s-t}\left(\mathcal{G}_{\lambda X Y}\right)\right) \mathrm{d} s .
$$

Proof. Relation (3.18) yields

$$
\left(\omega_{\lambda X Y}\left(\tau_{\lambda}^{t}(A)\right)-\omega_{\lambda X Y}\left(\tau_{0}^{t}(A)\right)\right) \omega_{X Y}\left(\mathcal{G}_{\lambda X Y}\right)=\omega_{X Y}\left(\left(\tau_{\lambda}^{t}(A)-\tau_{0}^{t}(A)\right) \mathcal{G}_{\lambda X Y}\right) .
$$

Since $\omega_{X Y}$ is $\tau_{0}$-invariant we have

$$
\begin{aligned}
\omega_{X Y}\left(\left(\tau_{\lambda}^{t}(A)-\tau_{0}^{t}(A)\right) \mathcal{G}_{\lambda X Y}\right) & =\omega_{X Y}\left(\left(\tau_{0}^{-t} \circ \tau_{\lambda}^{t}(A)-A\right) \tau_{0}^{-t}\left(\mathcal{G}_{\lambda X Y}\right)\right) \\
& =\mathrm{i} \lambda \int_{0}^{t} \omega_{X Y}\left(\tau_{0}^{-s}\left(\left[V, \tau_{\lambda}^{s}(A)\right]\right) \tau_{0}^{-t}\left(\mathcal{G}_{\lambda X Y}\right) \mathrm{d} s\right. \\
& =\mathrm{i} \lambda \int_{0}^{t} \omega_{X Y}\left(\left[V, \tau_{\lambda}^{s}(A)\right] \tau_{0}^{s-t}\left(\mathcal{G}_{\lambda X Y}\right)\right) \mathrm{d} s,
\end{aligned}
$$

and (3.19) follows.

Lemma 3.7 For any $\Lambda>0$ there exist $\epsilon>0$ and $\delta>0$ such that the function

$$
(\lambda, X, Y) \mapsto \omega_{X Y}\left(\mathcal{G}_{\lambda X Y}\right),
$$

extends to an analytic function on $\mathbb{C} \times D_{\epsilon}$ which satisfies

$$
\inf _{\lambda \in R_{\Lambda, \delta},(X, Y) \in D_{\epsilon}}\left|\omega_{X Y}\left(\mathcal{G}_{\lambda X Y}\right)\right|>0 .
$$

Proof. Since $\omega_{X Y}\left(\mathcal{G}_{\lambda X Y}\right)=\left(\Omega, \pi_{X Y}\left(\mathcal{G}_{\lambda X Y}\right) \Omega\right)$, the first statement is a special case of Lemma 3.5. Since $\omega_{X Y}\left(\mathcal{G}_{\lambda X Y}\right)>0$ for $\lambda, X, Y$ real, by continuity (3.20) holds for $\epsilon$ and $\delta$ small enough.

Lemma 3.8 For any $\Lambda>0$ there exist $\epsilon>0$ and $\delta>0$ such that for all $t \in \mathbb{R}$ the functions

$$
(\lambda, X, Y) \mapsto \omega_{\lambda X Y}\left(\tau_{0}^{t}\left(\mathfrak{F}_{j}\right)\right),
$$

extend to analytic functions on $R_{\Lambda, \delta} \times D_{\epsilon}$ such that

$$
\sup _{\lambda \in R_{\Lambda, \delta},(X, Y) \in D_{\epsilon}, t \in \mathbb{R}}\left|\omega_{\lambda X Y}\left(\tau_{0}^{t}\left(\mathfrak{F}_{j}\right)\right)\right|<\infty .
$$


Proof. For $X, Y$ real,

$$
\omega_{\lambda X Y}\left(\tau_{0}^{t}\left(\mathfrak{F}_{j}\right)\right)=\frac{\left(\Omega, \pi_{X Y}\left(\mathfrak{F}_{j}\right) \pi_{X Y}\left(\tau_{0}^{-t}\left(\mathcal{G}_{\lambda X Y}\right)\right) \Omega\right)}{\omega_{X Y}\left(\mathcal{G}_{\lambda X Y}\right)} .
$$

This identity and Lemmas 3.3, 3.5, and 3.7 yield the statement.

Lemma 3.9 Let $0<\Lambda<\Lambda_{0}$ be given. Then there exists $\epsilon>0$ such that for all $A=a^{\#}\left(f_{1}\right) \cdots a^{\#}\left(f_{m}\right)$ with $f_{j} \in \mathcal{D}$, the map

$$
(t, \lambda, X, Y) \mapsto \pi_{X Y}\left(\left[V, \tau_{\lambda}^{t}(A)\right]\right) \in \mathcal{B}(\mathcal{H}),
$$

extends to a continuous function on $\mathbb{R}_{+} \times\{\lambda \in \mathbb{C}|| \lambda \mid<\Lambda\} \times D_{\epsilon}$ which is analytic in $(\lambda, X, Y)$ for fixed $t \in \mathbb{R}$. Moreover,

$$
\int_{0}^{\infty} \sup _{\lambda \in \mathbb{C},|\lambda|<\Lambda,(X, Y) \in D_{\epsilon}}\left\|\pi_{X Y}\left(\left[V, \tau_{\lambda}^{t}(A)\right]\right)\right\| \mathrm{d} t<\infty .
$$

Proof. The expansion (1.1) yields that

$$
\begin{aligned}
\pi_{X Y}\left(\left[V, \tau_{\lambda}^{t}(A)\right]\right) & =\pi_{X Y}\left(\left[V, \tau_{0}^{t}(A)\right]\right) \\
& +\sum_{n=1}^{\infty}(\mathrm{i} \lambda)^{n} \int_{0 \leq s_{n} \leq \cdots \leq s_{1} \leq t} \pi_{X Y}\left(\left[V,\left[\tau_{0}^{s_{n}}(V),\left[\cdots,\left[\tau_{0}^{s_{1}}(V), \tau_{0}^{t}(A)\right] \cdots\right]\right]\right]\right) \mathrm{d} s_{1} \cdots \mathrm{d} s_{n} .
\end{aligned}
$$

Set

$$
C_{X Y}^{(0)}=\pi_{X Y}\left(\left[V, \tau_{0}^{t}(A)\right]\right),
$$

and, for $n \geq 1$,

$$
C_{X Y}^{(n)}\left(t, s_{1}, \ldots, s_{n}\right)=\pi_{X Y}\left(\left[V,\left[\tau_{0}^{s_{n}}(V),\left[\cdots,\left[\tau_{0}^{s_{1}}(V), \tau_{0}^{t}(A)\right] \ldots\right]\right]\right]\right) .
$$

Theorem 1.1 yields that for each $n$ there exist a finite index set $\mathcal{P}_{n}(A)$, scalar functions $G_{A, p}^{(n)}$ which do not depend on $X, Y$, and monomials $F_{A, p}^{(n)} \in \mathcal{O}$ such that

$$
C_{X Y}^{(n)}\left(t, s_{1}, \ldots, s_{n}\right)=\sum_{p \in \mathcal{P}_{n}(A)} G_{A, p}^{(n)}\left(t, s_{1}, \ldots, s_{n}\right) \pi_{X Y}\left(F_{A, p}^{(n)}\right) .
$$

Recall our standing assumption (1.3) and Part (2) of Theorem 1.1. Let $\delta>0$ be such that

$$
C_{0} \equiv(1+\delta)^{2 \bar{n}-1}<\frac{\Lambda_{0}}{\Lambda}
$$

Applying Lemma 3.4 with this $\delta$ to the factors of $\pi_{X Y}\left(F_{A, p}^{(n)}\right)$ we conclude that there exists $\epsilon>0$ (which depends on $\delta$ ) such that for all $n$ the functions

$$
(X, Y) \mapsto \pi_{X Y}\left(F_{p}^{(n)}\right) \in \mathcal{B}(\mathcal{H}),
$$

extend to analytic functions on $D_{\epsilon}$ satisfying

$$
\sup _{t, s_{1}, \ldots s_{n} \in \mathbb{R},(X, Y) \in D_{\epsilon}}\left\|\pi_{X Y}\left(F_{p}^{(n)}\right)\right\| \leq C_{1} C_{0}^{n+1},
$$

where $C_{1}=(1+\delta)^{m-1}\left[\max \left(1,\left\|f_{1}\right\|, \cdots,\left\|f_{m}\right\|\right)\right]^{m-1}$. By Part (3) of Theorem 1.1,

$$
\sum_{n=0}^{\infty}|\Lambda|^{n+1} \mathrm{C}_{0}^{n+1} \sum_{p \in \mathcal{P}_{n}(A)} \int_{0 \leq s_{n} \leq \cdots \leq s_{1} \leq t<\infty}\left|G_{A, p}^{(n)}\left(t, s_{1}, \ldots, s_{n}\right)\right| \mathrm{d} t \mathrm{~d} s_{1} \cdots \mathrm{d} s_{n}<\infty
$$


and we conclude that

$$
\int_{0}^{\infty} \sup _{\lambda \in \mathbb{C},|\lambda|<\Lambda,(X, Y) \in D_{\epsilon}}\left\|\pi_{X Y}\left(\left[V, \tau_{\lambda}^{t}\left(\mathfrak{F}_{j}\right)\right]\right)\right\| \mathrm{d} t<\infty .
$$

We are now ready to complete:

Proof of Theorem 3.1. We start with formula (3.19). By Lemmas 3.7 and 3.8, it suffices to show that for some $\epsilon>0$ the functions

$$
(\lambda, X, Y) \mapsto \int_{0}^{t}\left(\Omega, \pi_{X Y}\left(\left[V, \tau_{\lambda}^{s}\left(\mathfrak{F}_{j}\right)\right]\right) \pi_{X Y}\left(\tau_{0}^{s-t}\left(\mathcal{G}_{\lambda X Y}\right)\right) \Omega\right) \mathrm{d} s
$$

extend to analytic functions on $\{\lambda \in \mathbb{C}|| \lambda \mid<\Lambda\} \times D_{\epsilon}$ such that

$$
\sup _{\lambda \in \mathbb{C},|\lambda|<\Lambda,(X, Y) \in D_{\epsilon}, t \geq 0}\left|\int_{0}^{t}\left(\Omega, \pi_{X Y}\left(\left[V, \tau_{\lambda}^{s}\left(\mathfrak{F}_{j}\right)\right]\right) \pi_{X Y}\left(\tau_{0}^{s-t}\left(\mathcal{G}_{\lambda X Y}\right)\right) \Omega\right) \mathrm{d} s\right|<\infty .
$$

By Lemma 3.5, it suffices to show that the functions

$$
(t, \lambda, X, Y) \mapsto \pi_{X Y}\left(\left[V, \tau_{\lambda}^{t}\left(\mathfrak{F}_{j}\right)\right]\right) \in \mathcal{B}(\mathcal{H})
$$

extend to continuous functions on $\mathbb{R}_{+} \times\{\lambda \in \mathbb{C}|| \lambda \mid<\Lambda\} \times D_{\epsilon}$ which, for fixed $t$, are analytic in $(\lambda, X, Y)$ and satisfy the bound

$$
\int_{0}^{\infty} \sup _{\lambda \in \mathbb{C},|\lambda|<\Lambda,(X, Y) \in D_{\epsilon}}\left\|\pi_{X Y}\left(\left[V, \tau_{\lambda}^{t}\left(\mathfrak{F}_{j}\right)\right]\right)\right\| \mathrm{d} t<\infty .
$$

By (A2) and (A3), every $\mathfrak{F}_{j}$ can be written as a finite sum of monomials $a^{\#}\left(f_{1}\right) \cdots a^{\#}\left(f_{m}\right)$ with $f_{k} \in \mathcal{D}$, and the result follows from Lemma 3.9.

\section{References}

[Ar] Araki, H.: Relative Hamiltonians for faithful normal states of a von Neumann algebra. Publ. R.I.M.S., Kyoto Univ. 9, 165 (1973).

[AJPP1] Aschbacher, W., Jakšić, V., Pautrat, Y., Pillet, C.-A.: Topics in non-equilibrium quantum statistical mechanics. In Open Quantum Systems III. Attal, S., Joye, A., Pillet, C.-A. editors. Lecture Notes in Mathematics 1882, Springer, New York (2006).

[AJPP2] Aschbacher, W., Jakšić, V., Pautrat, Y., Pillet, C.-A.: Transport properties of quasi-free Fermions. Preprint.

[AM] Aizenstadt, V.V., Malyshev, V.A.: Spin interaction with an ideal Fermi gas. J. Stat. Phys. 48, 51 (1987).

[AW] Araki, H., Wyss, W.: Representations of canonical anti-commutation relations. Helv. Phys. Acta 37, 136 (1964).

[BGM] Botvich, D.D., Gută, M., Maassen, H.: Stability of Bose dynamical systems and branching theory. Preprint (mp_arc 99-130).

[BM1] Botvich, D.D., Malyshev, V.A.: Unitary equivalence of temperature dynamics for ideal and locally perturbed Fermi gas. Commun. Math. Phys. 91, 301 (1983).

[BM2] Botvich, D.D., Malyshev, V.A.: Asymptotic completeness and all that for an infinite number of fermions. In ManyParticle Hamiltonians: Spectra and Scattering. Minlos, R. A. editor. Advances in Soviet Mathematics 5, 39, AMS, Providence (1991).

[BR1] Bratteli, O., Robinson, D. W.: Operator Algebras and Quantum Statistical Mechanics 1. Springer-Verlag, Berlin (1987).

[BR2] Bratteli, O., Robinson, D. W.: Operator Algebras and Quantum Statistical Mechanics 2. Second edition, SpringerVerlag, Berlin (1996). 
[Da] Davies, E.B.: Markovian master equations. Commun. Math. Phys. 39, 91 (1974).

[DJP] Dereziński, J., Jakšić, V., Pillet, C.-A.: Perturbation theory of $W^{*}$-dynamics, KMS-states and Liouvilleans. Rev. Math. Phys. 15, 447 (2003).

[FMU] Fröhlich, J., Merkli, M., Ueltschi, D.: Dissipative transport: thermal contacts and tunneling junctions. Ann. Henri Poincaré 4, 897 (2003).

[JKP] Jakšić, V., Kritchevski, E., Pillet, C.-A.: Mathematical theory of the Wigner-Weisskopf atom. In Large Coulomb Systems. Lecture Notes on Mathematical Aspects of QED. Derezinski, J. Siedentop, H. editors. Lecture Notes in Physics 695, Springer (2006).

[JOP1] Jakšić, V., Ogata, Y., Pillet, C.-A.: The Green-Kubo formula and the Onsager reciprocity relations in quantum statistical mechanics. Commun. Math. Phys. 265, 721 (2006).

[JOP2] Jakšić, V., Ogata, Y., Pillet, C.-A.: Linear response theory for thermally driven quantum open systems. J. Stat. Phys. 123, 547, (2006).

[JOP3] Jakšić, V., Ogata, Y., Pillet, C.-A.: The Green-Kubo formula for the spin-fermion system. Commun. Math. Phys. 268, 401 (2006).

[JP1] Jakšić, V., Pillet, C-A.: On entropy production in quantum statistical mechanics. Commun. Math. Phys. 217, 285 (2001).

[JP2] Jakšić, V., Pillet, C.-A.: Non-equilibrium steady states for finite quantum systems coupled to thermal reservoirs. Commun. Math. Phys. 226, 131 (2002).

[JP3] Jakšić, V., Pillet, C.-A.: Mathematical theory of non-equilibrium quantum statistical mechanics. J. Stat. Phys. 108, 787 (2002).

[JP4] Jakšić, V., Pillet, C.-A.: On the strict positivity of entropy production. Preprint 2006.

[JP5] Jakšić, V., Pillet, C.-A.: In preparation.

[JPP] Jakšić, V., Pautrat, Y., Pillet, C.-A.: In preparation.

[LeSp] Lebowitz, J., Spohn, H.: Irreversible thermodynamics for quantum systems weakly coupled to thermal reservoirs. Adv. Chem. Phys. 39, 109 (1978).

[Ro] Robinson, D.W.: Return to equilibrium. Commun. Math. Phys. 31, 171 (1973).

[Ru1] Ruelle, D.: Natural nonequilibrium states in quantum statistical mechanics. J. Stat. Phys. 98, 57 (2000).

[Ru2] Ruelle, D.: Entropy production in quantum spin systems. Commun. Math. Phys. 224, 3 (2001).

[TM] Tasaki, S., Matsui, T.: Fluctuation theorem, nonequilibrium steady states and MacLennan-Zubarev ensembles of a class of large quantum systems. Fundamental Aspects of Quantum Physics (Tokyo, 2001). QP-PQ: Quantum Probab. White Noise Anal., 17, 100. World Sci., River Edge NJ, (2003). 